\title{
Surface conduction of topological Dirac electrons in bulk insulating $\mathrm{Bi}_{2} \mathrm{Se}_{3}$
}

\author{
Dohun Kim ${ }^{1 \dagger}$, Sungjae Cho ${ }^{1 \dagger}$, Nicholas P. Butch ${ }^{1 *}$, Paul Syers ${ }^{1}$, Kevin Kirshenbaum ${ }^{1}$, Shaffique Adam ${ }^{2}$, \\ Johnpierre Paglione ${ }^{1}$ and Michael S. Fuhrer ${ }^{1 \star}$
}

\begin{abstract}
The newly discovered three-dimensional strong topological insulators (STIs) exhibit topologically protected Dirac surface states $^{1,2}$. Although the STI surface state has been studied spectroscopically, for example, by photoemission ${ }^{3-5}$ and scanned probes $^{6-10}$, transport experiments ${ }^{11-17}$ have failed to demonstrate the most fundamental signature of the STI: ambipolar metallic electronic transport in the topological surface of an insulating bulk. Here we show that the surfaces of thin $(\sim 10 \mathrm{~nm})$, low-doped $\mathrm{Bi}_{2} \mathrm{Se}_{3}\left(\approx 10^{17} \mathrm{~cm}^{-3}\right)$ crystals are strongly electrostatically coupled, and a gate electrode can completely remove bulk charge carriers and bring both surfaces through the Dirac point simultaneously. We observe clear surface band conduction with a linear Hall resistivity and a well-defined ambipolar field effect, as well as a charge-inhomogeneous minimum conductivity region ${ }^{18-20}$. A theory of charge disorder in a Dirac band ${ }^{19-21}$ explains well both the magnitude and the variation with disorder strength of the minimum conductivity ( 2 to $5 \mathrm{e}^{2} / h$ per surface) and the residual (puddle) carrier density $\left(0.4 \times 10^{12}\right.$ to $\left.4 \times 10^{12} \mathrm{~cm}^{-2}\right)$. From the measured carrier mobilities $320-1,500 \mathrm{~cm}^{2} \mathrm{~V}^{-1} \mathrm{~s}^{-1}$, the charged impurity densities $0.5 \times 10^{13}$ to $2.3 \times 10^{13} \mathrm{~cm}^{-2}$ are inferred. They are of a similar magnitude to the measured doping levels at zero gate voltage $\left(1 \times 10^{13}\right.$ to $\left.3 \times 10^{13} \mathrm{~cm}^{-2}\right)$, identifying dopants as the charged impurities.
\end{abstract}

$\mathrm{Bi}_{2} \mathrm{Se}_{3}$, as prepared, is observed to be n-type owing to $\mathrm{Se}$ vacancies. We find that mechanically exfoliated thin (thickness $t \approx 10 \mathrm{~nm}) \mathrm{Bi}_{2} \mathrm{Se}_{3}$ on $\mathrm{SiO}_{2} / \mathrm{Si}$ is invariably highly $\mathrm{n}$-doped with sheet charge densities $\gtrsim 10^{13} \mathrm{~cm}^{-2}$, much greater than expected considering the bulk charge density $\left(\approx 10^{17} \mathrm{~cm}^{-3}\right)$ in our low-doped starting material ${ }^{22}$, suggesting additional doping is induced by mechanical cleavage, reaction with ambient species ${ }^{14,23}$, or substrate interaction. To remove this doping, we employed two types of p-type doping schemes on mechanically exfoliated thin $\mathrm{Bi}_{2} \mathrm{Se}_{3}$ field-effect transistors on $300 \mathrm{~nm} \mathrm{SiO}_{2} / \mathrm{Si}$ back-gate substrates ${ }^{24-27}$ : (1) chemical doping with 2,3,5,6-tetrafluoro-7,7,8,8-tetracyanoquinodimethane (F4TCNQ) or (2) electrochemical doping with a polymer electrolyte top gate.

Figure la,b shows the schematics of the device structures and gating schemes. We exploit either the strong electron affinity $(\approx 5.4 \mathrm{eV})$ of $\mathrm{F} 4 \mathrm{TCNQ}$ molecules ${ }^{27}$ or the large capacitance $\left(\approx 1 \mu \mathrm{F} \mathrm{cm}^{-2}\right)$ of the electrochemical double layer at the interface between accumulated ions and the sample surface ${ }^{24-26}$ to induce negatively charged ions near the surface and p-type doping of $\mathrm{Bi}_{2} \mathrm{Se}_{3}$. In both cases the dopant density was fixed after cooling to cryogenic temperature, but further tuning of the carrier density was possible using the back gate (see Methods).

Figure $1 \mathrm{c}, \mathrm{d}$ shows the longitudinal resistivity $\rho_{\mathrm{xx}}$ and Hall carrier density $n_{\mathrm{H}}=1 /\left(e R_{\mathrm{H}}\right)$ (where $R_{\mathrm{H}}$ is the Hall coefficient and $e$ is the elementary charge) of a representative device (F4TCNQ-doped device 4) at various temperatures $T$ from 2 to $50 \mathrm{~K}$ as a function of the back-gate voltage $V_{\mathrm{g}}$. The plot of $\rho_{\mathrm{xx}}\left(V_{\mathrm{g}}\right)$ shows a peak at $V_{\mathrm{g}, 0} \approx-45 \mathrm{~V}$, and $n_{\mathrm{H}}$ changes sign at a similar $V_{\mathrm{g}}$, diverging positively (negatively) when approaching $V_{\mathrm{g}, 0}=-45 \mathrm{~V}$ from above (below). There is no evidence of an energy gap: $\rho_{\mathrm{xx}}(T)$ is metallic $\left(\mathrm{d} \rho_{\mathrm{xx}} / \mathrm{d} T>0\right)$ and saturates at low $T$, and $n_{\mathrm{H}}(T)$ shows little temperature dependence. The behaviour is strongly reminiscent of that seen for the two-dimensional Dirac electronic system in graphene ${ }^{18}$. Likewise, we identify the linear regions of $n_{\mathrm{H}}$ versus $V_{\mathrm{g}}$ for $V_{\mathrm{g}}>-35 \mathrm{~V}$ and $V_{\mathrm{g}}<-60 \mathrm{~V}$ as unipolar n- and p-doped regimes respectively, and the region $-35<V_{\mathrm{g}}<-60 \mathrm{~V}$ as an inhomogeneous regime where electron and hole transport are both present.

Figure 2a shows the Hall resistivity of device 4 as a function of magnetic field $\rho_{\mathrm{xy}}(B)$ at various gate voltages in the unipolar $\mathrm{n}$ - and p-doped regimes. The Hall resistivity in the unipolar regime is always linear over the entire range of magnetic field $( \pm 9 \mathrm{~T})$, indicating all bands contributing to the transport have similar mobility and the same carrier sign. Specifically, we can rule out the possibility of both bulk and surface channels participating in conduction (previously observed to give a nonlinear $\rho_{\mathrm{xy}}(B)$; refs $\left.11,12,28\right)$ or significant contribution to conduction by impurity bands, which should have much lower carrier mobilities (1-10 $\mathrm{cm}^{2} \mathrm{~V}^{-1} \mathrm{~s}^{-1}$; ref. 17). The measured linear ambipolar Hall effect, with carrier mobility of $>10^{3} \mathrm{~cm}^{2} \mathrm{~V}^{-1} \mathrm{~s}^{-1}$, is therefore a strong indication that conduction in our samples is dominated by the surface states.

We note that a previous work on single-gated $\mathrm{Bi}_{2} \mathrm{Se}_{3}$ of similar thickness, but heavily $(0.5 \%)$ Ca-doped ${ }^{17}$, also showed a superficially similar resistivity peak, interpreted there as the transition from bulk to surface conduction. No region of unipolar p-type Hall effect was observed. The authors concluded that significant band bending in these highly doped crystals led to very different carrier densities on either side of the device as well as an effective reduction of the bulk gap ${ }^{11,13,17}$. To determine whether band bending is important in our devices, we fabricated a further top gate on an F4TCNQ-doped device (device 5), using hydrogen silsequioxane (HSQ) as a top-gate dielectric.

Figure $2 \mathrm{~b}$ shows the resistivity $\rho_{\mathrm{xx}}$ of dual-gated device 5 as a function of applied displacement field to the top $\left(D_{\mathrm{tg}}\right)$ and bottom

\footnotetext{
${ }^{1}$ Center for Nanophysics and Advanced Materials, Department of Physics, University of Maryland, College Park, Maryland 20742-4111, USA, ${ }^{2}$ Center for Nanoscale Science and Technology, National Institute of Standards and Technology, Gaithersburg, Maryland 20899-6202, USA. †These authors contributed equally to this work. "Present addresses: Department of Physics and Materials Research Laboratory, University of Illinois at Urbana-Champaign, Urbana, Illinois 61801-2902, USA (S.C.); Condensed Matter and Materials Division, Lawrence Livermore National Laboratory, Livermore, California 94550, USA (N.P.B.). *e-mail: mfuhrer@umd.edu.
} 


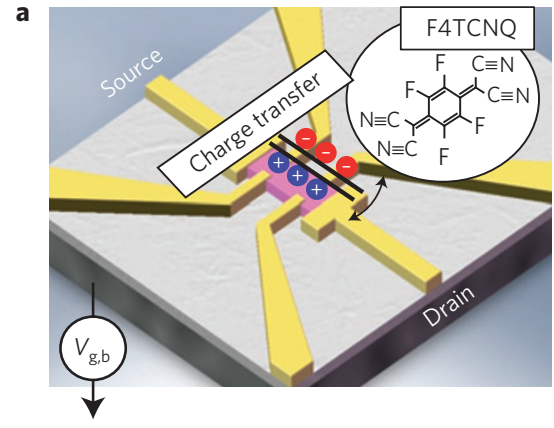

b

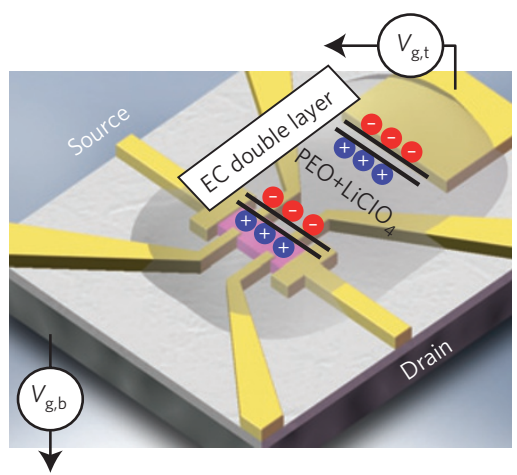

d

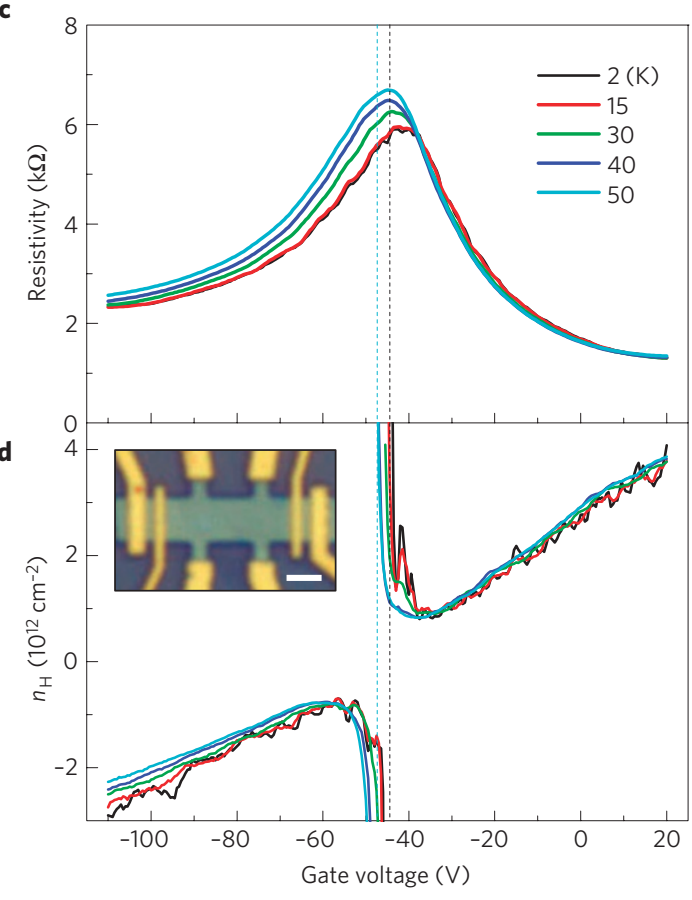

Figure $1 \mid \mathrm{Bi}_{2} \mathrm{Se}_{3}$ thin-film device. $\mathbf{a}, \mathbf{b}$, Schematics of the $\mathrm{p}$-type doping scheme and gate configuration for charge transfer doping with F4TCNQ organic molecules (a) and polymer electrolyte $\left(\mathrm{PEO}+\mathrm{LiClO}_{4}\right)(\mathbf{b})$ top gating. $\mathbf{c}, \mathbf{d}$, Longitudinal resistivity $\rho_{\mathrm{xx}}(\mathbf{c})$ and sheet carrier density $(\mathbf{d})$ determined from Hall measurement as a function of back-gate voltage for device 4 (F4TCNQ-doped) at various temperatures from 2 to $50 \mathrm{~K}$, as indicated in the figure. The inset of $\mathbf{d}$ shows an optical micrograph of the device. The scale bar is $2 \mu \mathrm{m}$.

$\left(D_{\mathrm{bg}}\right)$ surfaces (see Methods). The data are presented as a polar plot of the normalized resistivity $\left(\rho_{\mathrm{xx}} / \rho_{\max }\right)$ of the device as a function of total magnitude of displacement field $D_{\text {total }}=\left|D_{\mathrm{bg}}+D_{\mathrm{tg}}\right|$ and asymmetry factor, defined by $\alpha=(4 / \pi) \tan ^{-1}\left[\left(D_{\mathrm{tg}}-D_{\mathrm{bg}}\right) / D_{\text {total }}\right]$. We find that the measured resistivity depends only on the total displacement field, proportional to the total charge density in the $\mathrm{Bi}_{2} \mathrm{Se}_{3}$ slab. We conclude from the observed azimuthal symmetry that both surfaces are gated simultaneously with either gate and their chemical potential lies at the same level. If the gates acted independently on top and bottom surfaces, then the maximum resistivity peak associated with the transition from $n$ - to p-doping in each surface should broaden or split with increasing asymmetry; no such effect is observed. Remarkably, we find that simultaneous gating can be achieved even with a single-gate electrode $(\alpha= \pm 1)$. We ascribe this effect to (1) the strong electrostatic coupling of the surfaces due to the large inter-surface capacitance provided by the thin, lightly doped $\mathrm{Bi}_{2} \mathrm{Se}_{3}$, which has a high relative dielectric constant $\kappa \approx 100$; and (2) the low density of states of the Dirac surface. The net result is that the electrostatic inter-surface capacitance exceeds the quantum capacitance of each surface, in which case the two surface potentials become locked together (see Supplementary Information for a further discussion).

Having eliminated the possibilities of band bending or significant contribution to the conductivity by bulk or impurity states, we conclude that our measurement probes the conductance of the simultaneously gated ambipolar Dirac surfaces states. Our results therefore represent the first experimental demonstration of metallic, ambipolar, gapless electronic conduction of the topological surface state in $\mathrm{Bi}_{2} \mathrm{Se}_{3}$ in the absence of bulk carriers; the defining quality of a topological insulator.

Below, we analyse in more detail the transport properties of the topological surface state as a function of carrier density per surface $n$ estimated from $n=\left(C_{\mathrm{g}} / 2 e\right)\left(V_{\mathrm{g}}-V_{\mathrm{g}, 0}\right)$, where $V_{\mathrm{g}, 0}$ is the gate voltage at which $R_{\mathrm{H}}=0$, which corresponds closely to the gate voltage of minimum conductivity. Figure $3 \mathrm{a}-\mathrm{c}$ shows the conductivity per layer $(\sigma)$, the Hall carrier density per layer $\left(n_{\mathrm{H} \text { layer }}=1 / 2 R_{\mathrm{H}} e\right)$, and field effect mobility $\mu=\sigma / n e$ versus carrier density per layer $n$. Data are shown for devices 1-3, with electrolyte gating, and devices 4 and 5, charge-transfer-doped with F4TCNQ. Several features are notable immediately in Fig. 3 and comprise the main experimental observations in this work. On tuning the carrier density: (1) $\sigma$ and $n_{\mathrm{H} \text {,layer }}$ show clear ambipolar conduction with well-defined p- and n-regions, (2) $\left|n_{\mathrm{H} \text {,layer }}\right|$ shows a minimum value $\left(n^{*}\right)$ for p- and n-conduction, (3) $\sigma$ shows a roughly linear carrier density dependence for $n^{*}<n<n_{\text {bulk }}$, where $n_{\text {bulk }} \approx 5 \times 10^{12} \mathrm{~cm}^{-2}$ is the carrier density above which the bulk conduction band is expected to be populated, and (4) a minimum conductivity $\left(\sigma_{\min }=2 e^{2} / \mathrm{h}\right.$ to $\left.5 e^{2} / h\right)$ is observed.

Extending the theory of charge disorder in graphene ${ }^{19,20}$, a recent theoretical study predicts the conductivity as being limited by charged-impurity scattering in STI of the form (assuming a linear Dirac band $)^{21}$,

$$
\begin{aligned}
& \sigma(n) \sim C\left|\frac{n}{n_{\mathrm{imp}}}\right|\left[e^{2} / h\right] \quad \text { for } n>n^{*} \\
& \sigma(n) \sim C\left|\frac{n^{*}}{n_{\mathrm{imp}}}\right|\left[e^{2} / h\right] \quad \text { for } n<n^{*}
\end{aligned}
$$

where $n_{\text {imp }}$ is the charged impurity density, $C$ is a constant that depends on the Wigner-Seitz radius $r_{s}$, and $n^{*}$ is identified as the residual carrier density in electron and hole puddles. For $\mathrm{Bi}_{2} \mathrm{Se}_{3}$ on $\mathrm{SiO}_{2}$ (ref. 21) we expect $0.05<r_{s}<0.2$ and $30<C<300$. See Supplementary Information for a more detailed description of the theory. For $n^{*}<n<\approx 5 \times 10^{12} \mathrm{~cm}^{-2}$, we fit $\sigma(n)$ to equation (1a) (Fig. 3a, dashed lines), to obtain the field-effect mobility $\mu_{\mathrm{FE}}=\mathrm{Ce} / n_{\mathrm{imp}} h$ for each device. $\mu_{\mathrm{FE}}$ ranges from $320-1,500 \mathrm{~cm}^{2} \mathrm{~V}^{-1} \mathrm{~s}^{-1}$, reflecting different amounts of disorder in the samples. We identify the initial ntype dopants and defects induced by mechanical exfoliation as likely sources of the disorder. The decrease in $\mu_{\mathrm{FE}}$ with further electrolytic 


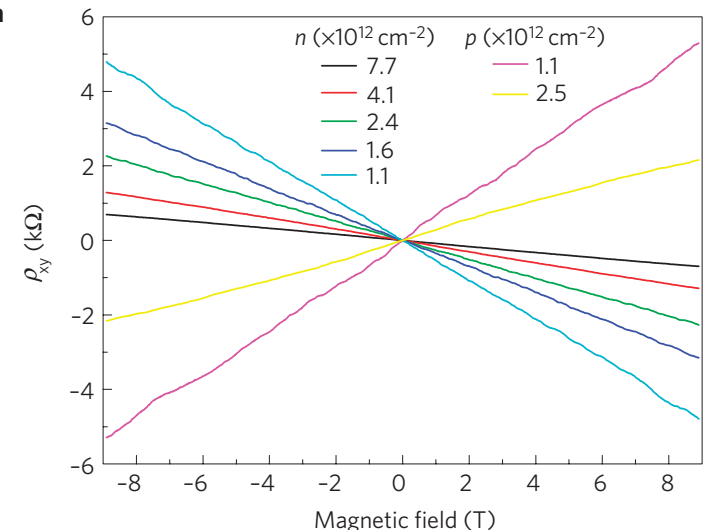

b

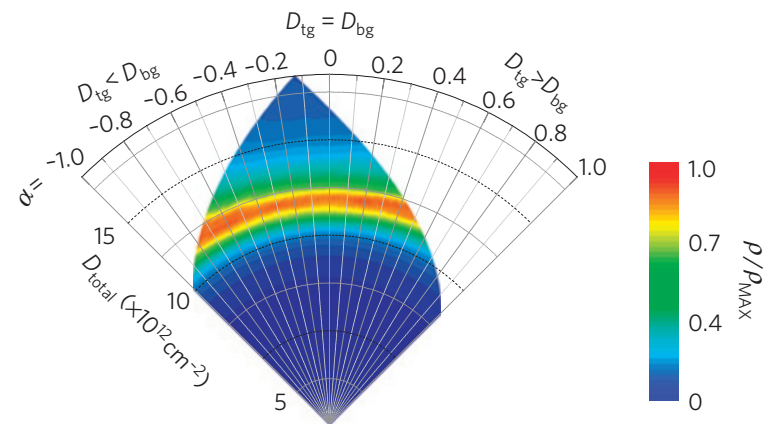

Figure 2 | Single band conduction in the topological insulator regime. a, Hall resistivity $\rho_{\mathrm{xy}}$ of device 4 as a function of magnetic field $B$ at a temperature of $2 \mathrm{~K}$ at different carrier densities tuned by the back-gate electrode. $\mathbf{b}$, Polar plot of the normalized longitudinal resistivity $\rho_{\mathrm{xx}}$ of the dual-gated $\mathrm{Bi}_{2} \mathrm{Se}_{3}$ thin-film device as a function of the total magnitude of displacement field $\left(D_{\text {total }}\right)$ and the gating asymmetry factor $\alpha$ (defined in the text).

gating (device 1 run 2) indicates electrochemical damage, probably solvation of Se ions. The observation of sublinear $\sigma(n)$ at $n<n_{\text {bulk }}$ in devices 3-5 may indicate that there are further types of disorder, for example neutral point defects, that should be considered.

The observed minimum conductivity of the Dirac electronic band can be understood well through equation (1b) as being due to the residual carrier density $n^{*}$ in electron and hole puddles induced by the charged impurity potential at nominally zero carrier density: $\sigma_{\min }=n^{*} e \mu$, where $n^{*}$ is calculated self-consistently ${ }^{20}$ as a function of $n_{\text {imp }}, r_{s}$ and $d$, the distance of the impurities to the Dirac surface. The self-consistent theory predicts that $n^{*}$ increases with increasing disorder and $\sigma_{\min }$ depends only weakly on disorder. Figure 4 shows the experimentally observed residual carrier density $n^{*}$ per surface for each device (Fig. 4a) as well as $\sigma_{\text {min }}$ per surface (Fig. $4 \mathrm{~b}$ ) as a function of the experimentally measured inverse mobility $1 / \mu_{\mathrm{FE}}$, which reflects the disorder strength. (For devices in which $n^{*}$ could be measured for $\mathrm{p}$ - and $\mathrm{n}$-type conduction, both values are shown.) The shaded regions reflect the expectations of the self-consistent theory using parameter ranges $0.05<r_{s}<0.2$ and $d=0.1 \AA-15 \AA$. We see a good agreement between experiment and theory in that (1) $\sigma_{\min }$ is weakly dependent on disorder strength $\left(1 / \mu_{\mathrm{FE}}\right)$ and (2) $n^{*}$ increases with disorder strength $\left(1 / \mu_{\mathrm{FE}}\right)$. Particularly for increasing disorder in the same device (Device 1 run 1 versus run 2), $n^{*}$ increases but $\sigma_{\min }$ is almost unchanged (arrows in Fig. 4a,b). The experimental data agree best with the upper range of the theoretical estimates, corresponding to small $d=0.1 \AA$ and large $r_{s}=0.2$. Assuming $r_{s}=0.2$, we infer an impurity density $n_{\text {imp }}$ ranging from $0.5 \times 10^{13}$ to $2.3 \times 10^{13} \mathrm{~cm}^{-2}$, much larger than for graphene exfoliated on similar $\mathrm{SiO}_{2}$ substrates ${ }^{29}$, but comparable to the observed initial doping level of $1 \times 10^{13}$ to $3 \times 10^{13} \mathrm{~cm}^{-2}$,

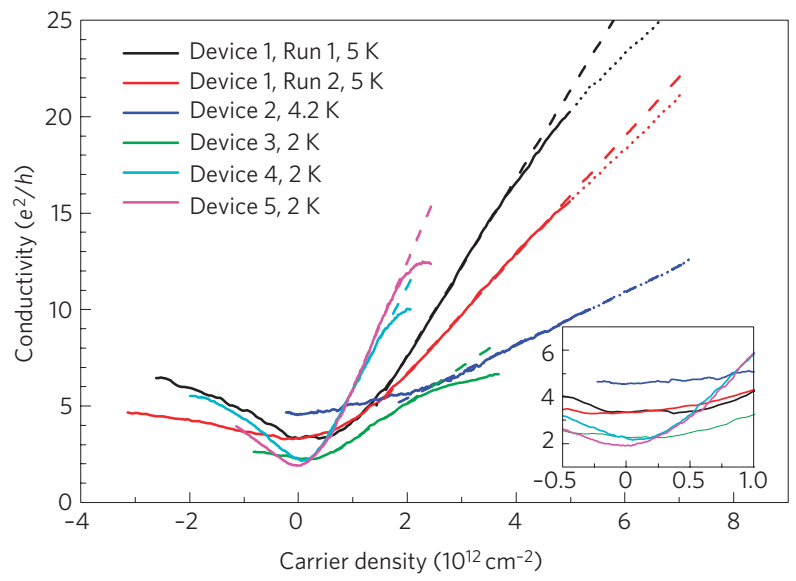

b

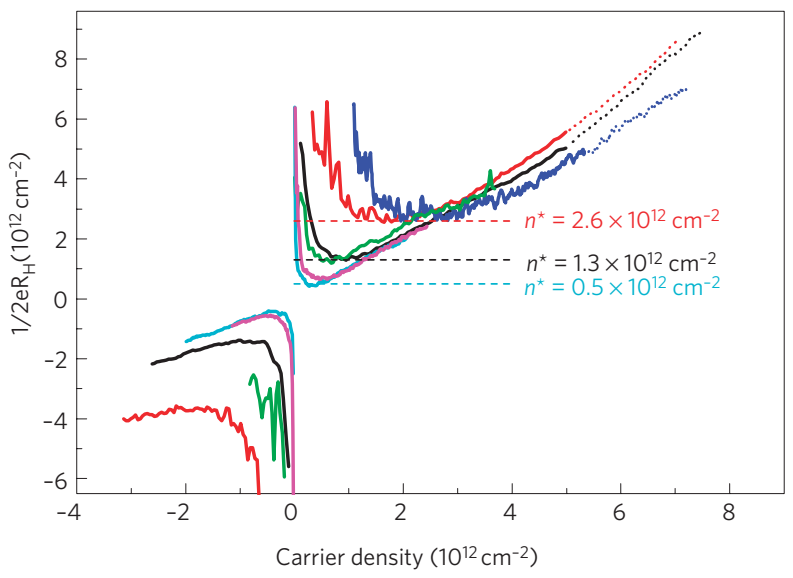

c

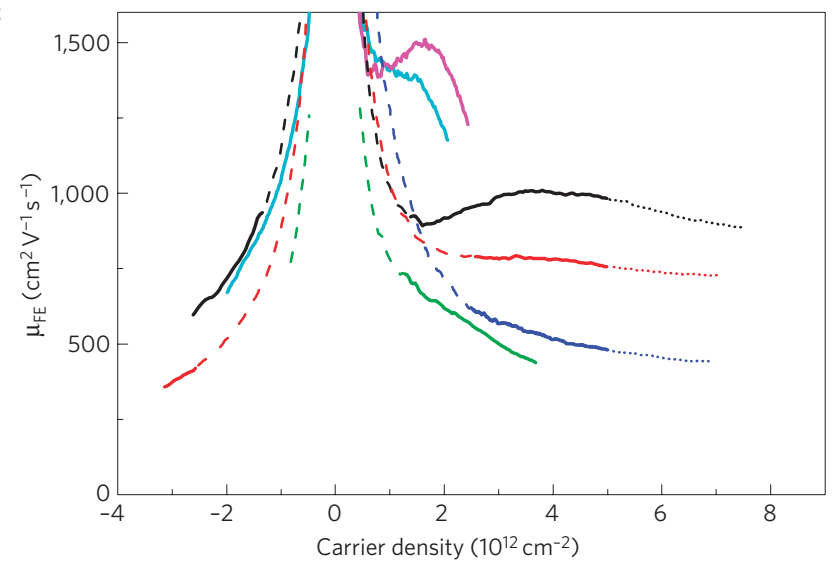

Figure 3 | Transport properties of the $\mathrm{Bi}_{2} \mathrm{Se}_{3}$ surface state. a, The conductivity per surface versus carrier density per surface $\sigma(n)$ at zero magnetic field for five different devices. Devices 1-3 are electrolyte-gated and devices 4 and 5 are F4TCNQ-doped. The inset shows $\sigma(n)$ near the Dirac point. Dashed lines are fits to equation (1a). Transport data outside the topological regime $\left(n>n_{\text {bulk }}=5 \times 10^{12} \mathrm{~cm}^{-2}\right)$ are denoted as dotted curves. b, Hall carrier density per surface versus carrier density measured at the same conditions as in $\mathbf{a}$. Dashed lines show the residual carrier density $n^{*}$ (defined in the text) for different devices. c, Variation of field effect mobility as a function of carrier density. Dashed curves indicate the region $|n|<n^{*}$ within which electron and hole puddles dominate transport.

suggesting that the dopants are the charged impurities responsible for limiting the mobility (see Supplementary Information).

The simple theory somewhat underestimates $n^{*}$ and $\sigma_{\min }$, but we expect that the theory can be refined to take into account the 


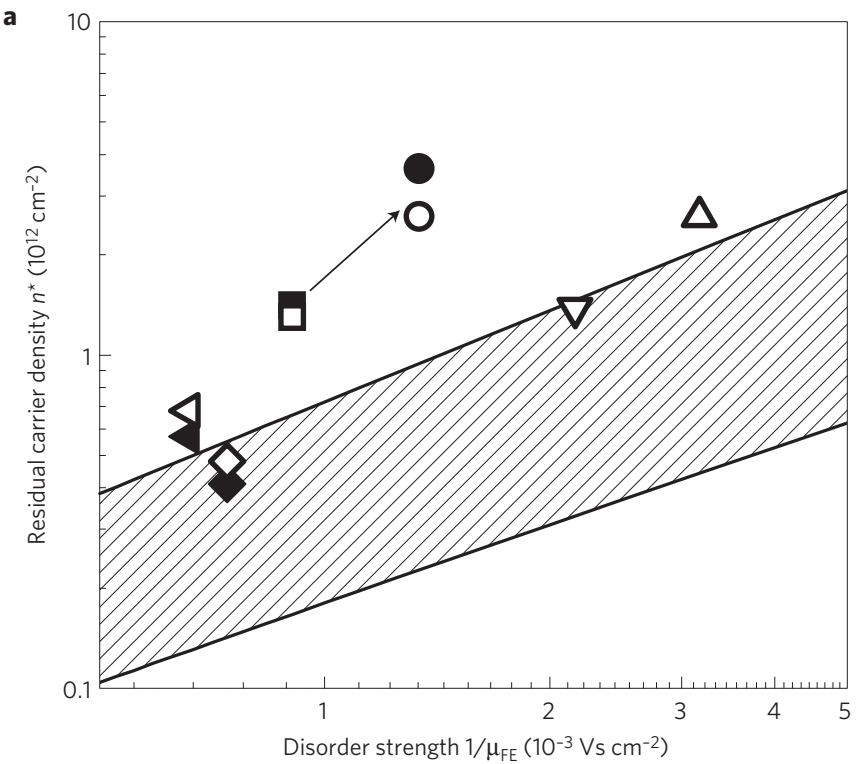

b

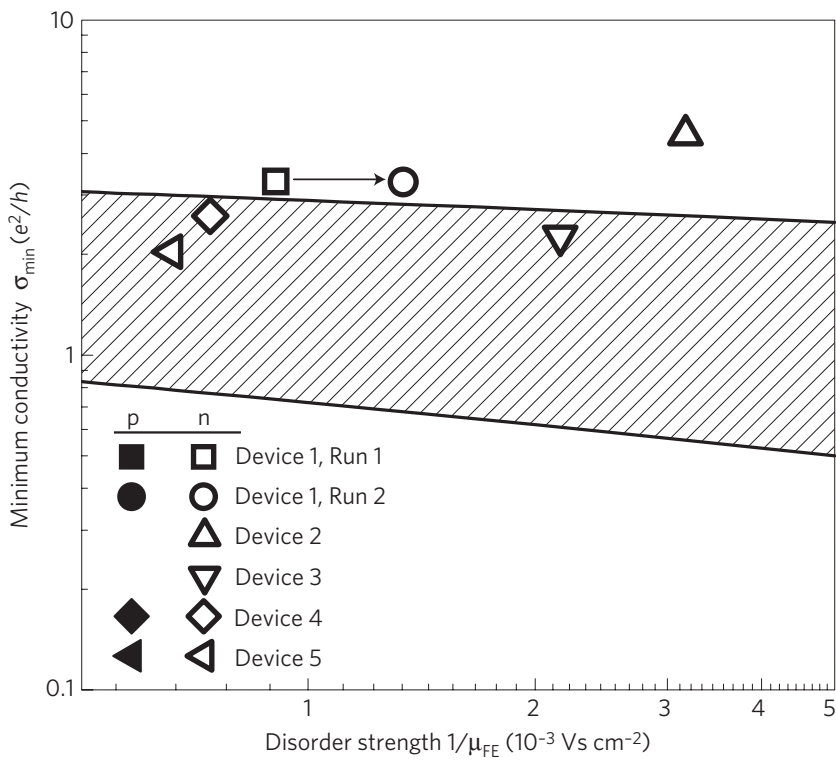

Figure 4 | Charge inhomogeneity and minimum conductivity versus disorder strength. a, Residual carrier density $n^{*}$ versus inverse field effect mobility $\left(1 / \mu_{\mathrm{FE}}\right)$. b. Minimum conductivity $\sigma_{\min }$ versus $1 / \mu_{\mathrm{FE}}$. Shaded areas indicate the expectations of the self-consistent theory of ref. 20, open symbols are experimental data.

nonlinearity and asymmetry of the $\mathrm{Bi}_{2} \mathrm{Se}_{3}$ surface state bands ${ }^{30}$. Notably, the larger Fermi velocity for the electron band would increase the conductivity above the estimate in equation (1) for $\mathrm{n}$-type conduction, indicating that the disorder strength is probably somewhat underestimated from the n-type mobility. Shifting the points to the right (to larger disorder strength) in Fig. 4 would indeed improve the agreement between experiment and theory.

Reducing the n-type doping of TI thin films by external agents provides an effective and simple way to probe topological surface transport properties in the absence of bulk conduction. For the present devices the level of charged impurity disorder is on the order of $\sim 10^{13} \mathrm{~cm}^{-2}$, limiting the mobility to $320-1,500 \mathrm{~cm}^{2} \mathrm{~V}^{-1} \mathrm{~s}^{-1}$. However, owing to the large dielectric constants in existing topological insulators, reduction of impurity concentrations to levels seen in the best bulk crystals $\left(<10^{17} \mathrm{~cm}^{-3}\right.$ corresponding to $<10^{11} \mathrm{~cm}^{-2}$ in a $10 \mathrm{~nm}$ thick crystal $^{22}$ ) would allow mobilities exceeding $10^{5} \mathrm{~cm}^{2} \mathrm{~V}^{-1} \mathrm{~s}^{-1}$. Hence understanding and eliminating the doping presently observed in all thin crystals and films is of central importance to increasing the mobility of the topological surface state.

Note added in proof: After submission of this work we became aware of a scanning tunnelling microscopy study ${ }^{10}$ that directly observed the screened potential fluctuations caused by charged impurity disorder in $\mathrm{Bi}_{2} \mathrm{Se}_{3}$ and $\mathrm{Bi}_{2} \mathrm{Te}_{3}$, consistent with our interpretation of the minimum conductivity arising from charge inhomogeneity.

\section{Methods}

$\mathrm{Bi}_{2} \mathrm{Se}_{3}$ thin crystals were produced by micro-mechanical cleavage of bulk $\mathrm{Bi}_{2} \mathrm{Se}_{3}$ single crystals and deposited on doped $\mathrm{Si}$ covered with $300 \mathrm{~nm} \mathrm{SiO}{ }_{2}$. Thin crystals (thickness about $10 \mathrm{~nm}$ ) were identified using atomic force microscopy (AFM). The thin film was patterned in a Hall bar geometry using an Ar plasma at a pressure of $\approx 6.7 \mathrm{~Pa}\left(5 \times 10^{-2}\right.$ torr $)$. Au/Cr electrodes were defined by electron-beam lithography (see inset of Fig. $1 \mathrm{~d}$ ). A brief $(\approx 10 \mathrm{~s})$ selective surface treatment of the contact area with a $\mathrm{N}_{2}$ or Ar plasma before the deposition of metals was used to enhance the ohmic conduction of the contacts.

The p-type doping for devices 1-3 was achieved by applying a negative voltage to a polymer electrolyte consisting of $\mathrm{LiClO}_{4}$ and polyethylene oxide (PEO) in the weight ratio 0.12:1, as previously used for carbon nanotubes and graphene devices ${ }^{24-26}$. Molecular charge transfer doping for device 4 and 5 was done by thermal evaporation of $\approx 15 \mathrm{~nm}$ of F4TCNQ molecules (Aldrich) on top of the samples ${ }^{27}$. The devices were subsequently cooled down and further tuning of carrier density was done by sweeping the back-gate voltage at cryogenic temperature. For electrolyte-gated measurements, the samples were cooled down to $250 \mathrm{~K}$ in less than $1 \mathrm{~min}$ after applying the top-gate voltage to minimize electrochemical reaction $\mathrm{s}^{25}$.

As well as single-gated samples, we fabricated dual-gated samples based on F4TCNQ-doped samples (Fig. 2b). $60 \mathrm{~nm}$ of hydrogen silsequioxane (HSQ, XR-1541, Dow Corning) was spin-coated on an F4TCNQ-coated $\mathrm{Bi}_{2} \mathrm{Se}_{3}$ device and a top-gate electrode was defined by electron-beam lithography. We found that further fabrication on pre-doped devices increased the n-type doping level (for example, $\approx 1.2 \times 10^{13} \mathrm{~cm}^{-2}$ at zero gate field in Fig. $2 \mathrm{~b}$ compared with $\approx 0.3 \times 10^{13} \mathrm{~cm}^{-2}$ in Fig. 1d). From the Hall carrier density versus gate voltage, the bottom-gate and top-gate capacitances were determined to be $\approx 11 \mathrm{nF} \mathrm{cm}{ }^{-2}$ and $\approx 33 \mathrm{nF} \mathrm{cm}{ }^{-2}$, respectively; reasonable values considering the dielectric constants of $\mathrm{SiO}_{2}(\kappa \approx 3.9)$ and HSQ $(\kappa \approx 3)$.

Four-probe measurements of longitudinal and transverse electrical resistances were conducted using Stanford Research Systems SR830 Lock-in amplifiers and a commercial cryostat equipped with $9 \mathrm{~T}$ superconducting magnet. The Hall voltage was recorded in both polarities of the magnetic field $( \pm 1 \mathrm{~T})$ and anti-symmetrized to remove longitudinal voltage components. In the transport experiments a small and reproducible hysteresis in $V_{\mathrm{g}, 0}(\approx 2 \mathrm{~V})$ was observed during forward and backward gate voltage scans. As a consequence, resistivity and Hall data with the same $V_{\mathrm{g}}$ scan directions were compared in this work. Best fits to equation (1a) were determined using a least squares linear fit to $\sigma(n)$ in the linear regime, determined by identifying the region of roughly constant slope $\mathrm{d} \sigma / \mathrm{d} n$. Thermal runs as described here were performed for more than ten different $\mathrm{Bi}_{2} \mathrm{Se}_{3}$ samples of similar thickness, with qualitatively consistent results.

Note: certain commercial equipment, instruments or materials are identified in this paper to specify the experimental procedure adequately. Such an identification is not intended to imply any recommendation or endorsement by the National Institute of Standards and Technology, nor is it intended to imply that the materials or equipment identified are necessarily the best available for the purpose.

Received 25 August 2011; accepted 9 March 2012; published online 15 April 2012

\section{References}

1. Fu, L., Kane, C. L. \& Mele, E. J. Topological insulators in three dimensions. Phys. Rev. Lett. 98, 106803 (2007)

2. Zhang, H. J. et al. Topological insulators in $\mathrm{Bi}_{2} \mathrm{Se}_{3}, \mathrm{Bi}_{2} \mathrm{Te}_{3}$ and $\mathrm{Sb}_{2} \mathrm{Te}_{3}$ with a single Dirac cone on the surface. Nature Phys. 5, 438-442 (2009).

3. Hsieh, D. et al. A tunable topological insulator in the spin helical Dirac transport regime. Nature 460, 1101-1105 (2009).

4. Chen, Y. L. et al. Experimental realization of a three-dimensional topological insulator $\mathrm{Bi}_{2} \mathrm{Te}_{3}$. Science 325, 178-181 (2009).

5. Xia, Y. et al. Observation of a large-gap topological-insulator class with a single Dirac cone on the surface. Nature Phys. 5, 398-402 (2009).

6. Zhang, T. et al. Experimental demonstration of topological surface states protected by time-reversal symmetry. Phys. Rev. Lett. 103, 266803 (2009).

7. Alpichshev, Z. et al. STM imaging of electronic waves on the surface of $\mathrm{Bi}_{2} \mathrm{Te}_{3}$ : Topologically protected surface states and hexagonal warping effects. Phys. Rev. Lett. 104, 016401 (2010). 
8. Hanaguri, T. et al. Momentum-resolved Landau-level spectroscopy of Dirac surface state in $\mathrm{Bi}_{2} \mathrm{Se}_{3}$. Phys. Rev. B 82, 081305R (2010).

9. Cheng, P. et al. Landau quantization of topological surface states in $\mathrm{Bi}_{2} \mathrm{Se}_{3}$. Phys. Rev. Lett. 105, 076801 (2010).

10. Beidenkopf, H. et al. Spatial fluctuations of helical Dirac fermions on the surface of topological insulators. Nature Phys. 7, 939-943 (2011).

11. Steinberg, H., Gardner, D. R., Lee, Y. S. \& Jarillo-Herrero, P. Surface state transport and ambipolar electric field effect in $\mathrm{Bi}_{2} \mathrm{Se}_{3}$ nanodevices. Nano Lett. 10, 5032-5036 (2010).

12. Qu, D., Hor, Y. S., Xiong, J., Cava, R. J. \& Ong, N. P. Quantum oscillations and Hall anomaly of surface states in the topological insulator $\mathrm{Bi}_{2} \mathrm{Te}_{3}$. Science 329, 821-824 (2010).

13. Xiu, F. et al. Manipulating surface states in topological insulator nanoribbons. Nature Nanotech. 6, 216-221 (2011).

14. Analytis, J. G. et al. Two-dimensional surface state in the quantum limit of a topological insulator. Nature Phys. 6, 960-964 (2010).

15. Peng, H. et al. Aharonov-Bohm interference in topological insulator nanoribbons. Nature Mater. 9, 225-229 (2010).

16. Chen, J. et al. Gate-voltage control of chemical potential and weak antilocalization in $\mathrm{Bi}_{2} \mathrm{Se}_{3}$. Phys. Rev. Lett. 105, 176602 (2010).

17. Checkelsky, J. G., Hor, Y. S., Cava, R. J. \& Ong, N. P. Surface state conduction observed in voltage-tuned crystals of the topological insulator $\mathrm{Bi}_{2} \mathrm{Se}_{3}$. Phys. Rev. Lett. 106, 196801 (2010).

18. Novoselov, K. S. et al. Two-dimensional gas of massless Dirac fermions in graphene. Nature 438, 197-200 (2005).

19. Hwang, E. H., Adam, S. \& Das Sarma, S. Carrier transport in two-dimensional graphene layers. Phys. Rev. Lett. 98, 186806 (2007).

20. Adam, S., Hwang, E. H., Galitski, V. M. \& Das Sarma, S. A self-consistent theory for graphene transport. Proc. Natl Acad. Sci. USA 104, 18392-18397 (2007).

21. Culcer, D., Hwang, E. H., Stanescu, T. D. \& Das Sarma, S. Two-dimensional surface charge transport in topological insulators. Phys. Rev. B 82, 155457 (2010)

22. Butch, N. P. et al. Strong surface scattering in ultrahigh-mobility $\mathrm{Bi}_{2} \mathrm{Se}_{3}$ topological insulator crystals. Phys. Rev. B 81, 241301 (2010).

23. Kong, D. et al. Rapid surface oxidation as a source of surface degradation factor for $\mathrm{Bi}_{2} \mathrm{Se}_{3}$. ACS Nano 5, 4698-4703 (2011).

24. Das, A. et al. Monitoring dopants by Raman scattering in an electrochemically top-gated graphene transistor. Nature Nanotech. 3, 210-215 (2008).
25. Efetov, D. K. \& Kim, P. Controlling electron-phonon interactions in graphene at ultrahigh carrier densities. Phys. Rev. Lett. 105, 256805 (2010).

26. Lu, C. G., Fu, Q., Huang, S. M. \& Liu, J. Polymer electrolyte-gated nanotube field-effect carbon transistor. Nano Lett. 4, 623-627 (2004).

27. Coletti, C. et al. Charge neutrality and band-gap tuning of epitaxial graphene on SiC by molecular doping. Phys. Rev. B 81, 235401 (2010).

28. Bansal, M., Kim, Y. S., Brahlek, M., Eliav, E. \& Oh, S. Thickness-independent surface transport channel in topological insulator $\mathrm{Bi}_{2} \mathrm{Se}_{3}$ thin films. Preprint at http://arxiv.org/abs/1104.5709 (2011).

29. Chen, J. H. et al. Charged impurity scattering in graphene. Nature Phys. 4, 377-381 (2008).

30. Adam, S., Hwang, E. H. \& Sarma, S. D. 2D transport and screening in topological insulator surface states. Preprint at http://arxiv.org/abs/ 1201.4433 (2012).

\section{Acknowledgements}

The study of electronic transport in novel materials during electrochemical modification is supported as part of the Science of Precision Multifunctional Nanostructures for Electrical Energy Storage, an Energy Frontier Research Center funded by the US Department of Energy, Office of Science, Office of Basic Energy Sciences under Award Number DESC0001160. Additional support was provided by the National Science Foundation (NSF) DMR-1105224. Preparation of $\mathrm{Bi}_{2} \mathrm{Se}_{3}$ was supported by NSF MRSEC (DMR-0520471) and Defense Advanced Research Projects Agency (DARPA) MTO award (N66001-09-c-2067). N.P.B. was partially supported by the Center for Nanophysics and Advanced Materials. The authors acknowledge useful conversations with S. Das Sarma, E. Hwang and D. Culcer.

\section{Author contributions}

D.K. conceived the p-type doping schemes. D.K. and S.C. fabricated devices, performed the electrical measurements with K.K. and analysed the data. N.P.B., P.S. and J.P. prepared single crystal $\mathrm{Bi}_{2} \mathrm{Se}_{3}$ starting material. S.A. assisted with the theoretical analysis. D.K., S.C. and M.S.F. wrote the manuscript.

\section{Additional information}

The authors declare no competing financial interests. Supplementary information accompanies this paper on www.nature.com/naturephysics. Reprints and permissions information is available online at www.nature.com/reprints. Correspondence and requests for materials should be addressed to M.S.F. 